\title{
EMT or EMT-promoting transcription factors, where to focus the light?
}

\section{Stéphane Ansieau ${ }^{1,2,3,4,5}{ }^{*}$, Guillaume Collin ${ }^{1,2,3,4,5}$ and Louise Hill ${ }^{1,2,3,4,5}$}

1 INSERM UMR-S1052, Centre de Recherche en Cancérologie de Lyon, Lyon, France

${ }^{2}$ CNRS UMR5286, Centre de Recherche en Cancérologie de Lyon, Lyon, France

${ }^{3}$ LabEX DEVweCAN, Lyon, France

${ }^{4}$ UNIV UMR1052, Lyon, France

${ }^{5}$ Centre Léon Bérard, Lyon, France

*Correspondence: stephane.ansieau@lyon.unicancer.fr

Edited by:

Pierre Savagner, Institut National de la Santé et de la Recherche Médicale, France

Reviewed by:

Antonio Garcia De Herreros, Institut Hospital del Mar d'Investigacions Mèdiques (IMIM), Spain

Keywords: EMT, escape from fail-safe programs, EMT-TFs, plasticity, EMT-promoting

The epithelial-to-mesenchymal transition (EMT) is commonly considered as a main driving force of the metastatic cascade. An impressive series of experiments and observations worldwide supports its pivotal role in promoting cancer cell dissemination at the invasive fronts of tumors, intravasation, cell survival in fluids, and extravasation, with secondary site colonization being the only step requiring a return to an epithelial phenotype (1). Beyond this, commitment of epithelial cells into an EMT program has been associated with resistance to chemo, radio-, and hormono-therapies, definitively extending the interest of studying EMT from the embryologist community to the oncology and medical fields. Obviously, EMT turns out to constitute a transdifferentiation program, allowing epithelial cells to escape from numerous stresses, including mechanical constrains, hypoxia, nutrient depletion, and unfortunately therapeutic treatments (1). EMT is orchestrated by a restricted number of transcription factors mainly the three Snail, Twist, and Zeb families (EMT-TFs). With inclusion of miRNAs, these factors constitute a complex interactome, able to sensor multiple signals received from the proximal microenvironment and relay them onto gene expression $(2,3)$.

Generally undetectable in adult epithelial cells in homeostatic conditions, EMTTFs were found to be recurrently expressed in various types of cancers including multiple carcinomas, an expression often associated with a high metastatic risk. An outstanding amount of work has been performed over the last years evaluating their relative contribution to the initiation and maintenance of the EMT process, with a significant impact on the prognostic value occurring following to their detection in primary tumors or in disseminated cancer cells $(4,5)$. Does this mean that the oncogenic potential of these factors in epithelial cells only rely on their ability to promote EMT or does their oncogenic activity extend further than EMT induction?

By performing in vitro functional assays, we and others firstly demonstrated that several of these embryonic transcription factors, including the TWIST proteins, alleviated fail-safe program induction. Through this, they were shown to cooperate with mitogenic oncoproteins such as RAS and N-MYC in promoting cell transformation in vitro, as well as in lung and breast carcinoma development in transgenic mouse models (4, 6-9). The underlying mechanisms have been largely explored. The TWIST1 protein was found to directly interact with p53 and to destabilize the oncosuppressive protein by altering specific post-translational modifications (10). Furthermore, TWIST1 alleviates induction of cyclin-dependent kinase inhibitors (CDKN1A,CDKN2A, and $C D K N 2 B)$, thereby sustaining cell proliferation $(8,11)$. These pleiotropic properties probably provide TWIST proteins unique properties. As TWIST proteins experimentally are inefficient in triggering EMT as compared with SNAIL and ZEB proteins, their main functions may consist in protecting cells from fail-safe programs during the EMT-associated genetic reprograming. In this respect, they might be considered as survival factors rather than EMT inducers. Although SNAIL and ZEB proteins, unlike the TWIST transcription factors, fail to prevent HRAS ${ }^{\mathrm{G} 12 \mathrm{~V}}$. induced senescence in murine embryonic fibroblasts (B. Gras, unpublished data), we obviously cannot exclude that they facilitate the escape from oncogene-induced fail-safe programs in other cellular settings and/or experimental conditions. In support of this assumption, ZEB proteins were indeed reported to protect lung cancer cells from EGFR-induced senescence through their ability to down-modulate $C D K N 1 A$ and $C D K N 2 B$ (12). In line with this observation, ZEB1 was demonstrated to be positioned downstream of $\mathrm{RB}$ and to contribute to fibroblast immortalization induced by RB and RB-like protein depletion (13). Enforced expression of SNAIL or ZEB proteins in non-transformed mammary epithelial cells, and the consequent activation of RAS-downstream pathways, predominantly triggers EMT. Nonetheless, it also accidentally promotes the commitment of cells into a senescence program, unveiled by their SA- $\beta$-galactosidase activity (B Gras and SA, unpublished data). This observation is consistent with the reported anti-proliferative properties of SNAIL and ZEB proteins in epithelial cells (14) and with the recognized antagonism between cell proliferation and dissemination. It additionally gives a rationale to the restricted staining of these transcription factors to the tumor-stromal interface, stabilized by microenvironmental EMTpermissive conditions (15-17). The need to maintain ZEB and SNAIL proteins at 
a basal level to sustain epithelial cell proliferation is difficult to reconcile with a role in fail-safe program escape. Nonetheless, the fact that the EMT-promoting and fail-safe program inhibition induced by ZEB1 requires different levels of protein expression (18) suggests that a low protein level (and likely not always detectable by immunohistochemistry) is not incompatible with such a function. Most of these transcription factors are particularly unstable, subjected to post-translational modifications and thereby transiently stabilized and activated. Knockdown experiments, rather than stable enforced expression, are thus warranted to gain further insight into their functions. Such an approach has successfully been employed to emphasize the temporally distinct functions of SNAIL1 and TWIST1 during the TGF $\beta$-driven EMT (19). Interestingly, human sarcomas were recently shown to display high SNAIL1 expression and SNAIL1 was demonstrated to control the tumorigenic properties of mesenchymal cells (20). In this tumor progression model, the anti-apoptotic properties of SNAIL proteins may provide cells a survival advantage, which would enhance their potential to undergo neoplastic transformation. Additionally, the SNAIL1 protein has been reported to alleviate the differentiation of multipotent mesenchymal stem cells (21), the cells of origin of certain sarcomas [reviewed in Ref. (22)].

By facilitating the escape from fail-safe programs, TWIST proteins may not only contribute to facilitate tumor initiation but also provide cancer cells with proliferation and survival advantages. Obviously, numerous cancer cell lines from various tumor types including breast and lung carcinoma, sarcoma, and neuroblastoma were found to remain dependent on TWIST1 for their survival $(7,8,11)$. As already mentioned, ZEB1 was similarly shown to abrogate latent EGFR-induced senescence in lung carcinoma cells (12). The addiction to a specific embryonic transcription factor may be determined by the nature of the original insult, e.g., in murine pancreatic epithelial cells, TWIST1 is induced in response to K-RAS activation and, avoids replicative senescence by turning-down Cdkn2A (23).

As an interconnected transcriptional network, expression of SNAIL, TWIST, and ZEB proteins induces a profound genetic reprograming of cells, with the corresponding consequences upon epithelial integrity undoubtedly constituting only a single facet of this remodeling. A brief overview of the induced genetic changes unambiguously highlights profound metabolic modifications and in support of this observation, SNAIL1 was shown to favor glycolysis, glucose uptake, maintenance of ATP production in hypoxic conditions and to reduce ROS production $(24,25)$. An additional consequence of this genetic reprograming is to afford cells a "plastic" configuration, with an exacerbated adaptability to hostile environments and an ability to quickly respond to their needs. As an example, enforced expression of TWIST1 in mammary epithelial cells poorly impacts on cell morphology but significantly accelerates their commitment to EMT when submitted to TGF $\beta$, an EMT-promoting cytokine (9). Cell plasticity similarly determines the ability of EMT-committed cells to return to an epithelial phenotype in a restrictive microenvironment, promoting their capability to colonize secondary sites $(26,27)$. In this regard, neither epithelial nor mesenchymal cells, the two end points of the process, are likely to constitute the most aggressive cells, with the partially reprogramed and semi-committed cells being the most likely to switch between an invasive and proliferative status.

Partial reprograming driven by the embryonic transcription factors likely places cells at the intersection of different destinies, their outcomes being likely dictated by intrinsic properties, and/or genetic events. When combined with key regulators of cell determination, such as the SOX9 transcription factors, cells further commit to a dedifferentiation process (28). Dedifferentiation also takes place, at least to some extent, when the embryonic transcription factors are combined with mitogenic activations, leading to the reacquisition of some stem-cell-like properties, including a self-renewal potential $(9,29)$. In support of this assumption, combined expression of TWIST1 and an activated version of RAS in murine luminal committed mammary epithelial cells invariably leads to the development of carcinomas of a particular subtype referred as "claudin-low" (9): a group of tumors with enriched EMT and stem-cell features and originally believed to arise from mammary stem cells (30). The link between embryonic transcription factors and stemness has been further exemplified by the detection of ZEB1 specifically in poorly differentiated pancreatic carcinomas and the demonstration of its role in maintaining stemness through repression of stemness-inhibiting miRNAs (31). Combined EMT and stemness induction at the invasive fronts of tumors has been proposed as a first rationale to explain the dissemination of single cancer stem cells, able to colonize distant sites and yield secondary tumors with full heterogeneity (32).

Strikingly, partial commitment into EMT (and presumably the transition to this plastic state) was also demonstrated as sufficient to accelerate epithelial cell transformation. Presumably, the genetic reprograming impacts on multiple mitogenic (e.g., activation of the RAS pathway) and oncosuppressive (e.g., downmodulation of the activity of the phosphatase PP2A) pathways $(9,33)$. Whether cell dedifferentiation contributes to the oncogenic properties of these embryonic transcription factors in non-epithelial cells remains poorly investigated, with the exception of melanocytes. These neuralcrest derived cells endogenously express SNAIL2 and ZEB2, both of which activate MITF transcription and induce downstream target genes to promote cell survival and proliferation. Following activation of the NRAS/BRAF pathway, a driver mutation in melanomagenesis, a redistribution of the embryonic transcription factors takes place, with SNAIL2 and ZEB2 being replaced by ZEB1 and TWIST1. These two embryonic transcription factors display opposite functions to SNAIL2 and ZEB2, by turning-down MITF expression and silencing the downstream differentiation program to rather favor cell migration (34, 35). Strikingly, modulation of the MITF rheostat is determinant for melanocyte transformation (36). The reversible redistribution of these embryonic transcription factors furthermore regulates the equilibrium between the proliferative and invasive states of melanoma cancer cells, and thereby dictates their ability to complete the metastatic process. In support of this expectation, ZEB2 was identified as essential for secondary site colonization (37). It is actually very likely that the ability to alleviate differentiation programs or to induce cell dedifferentiation will turn, in the near 


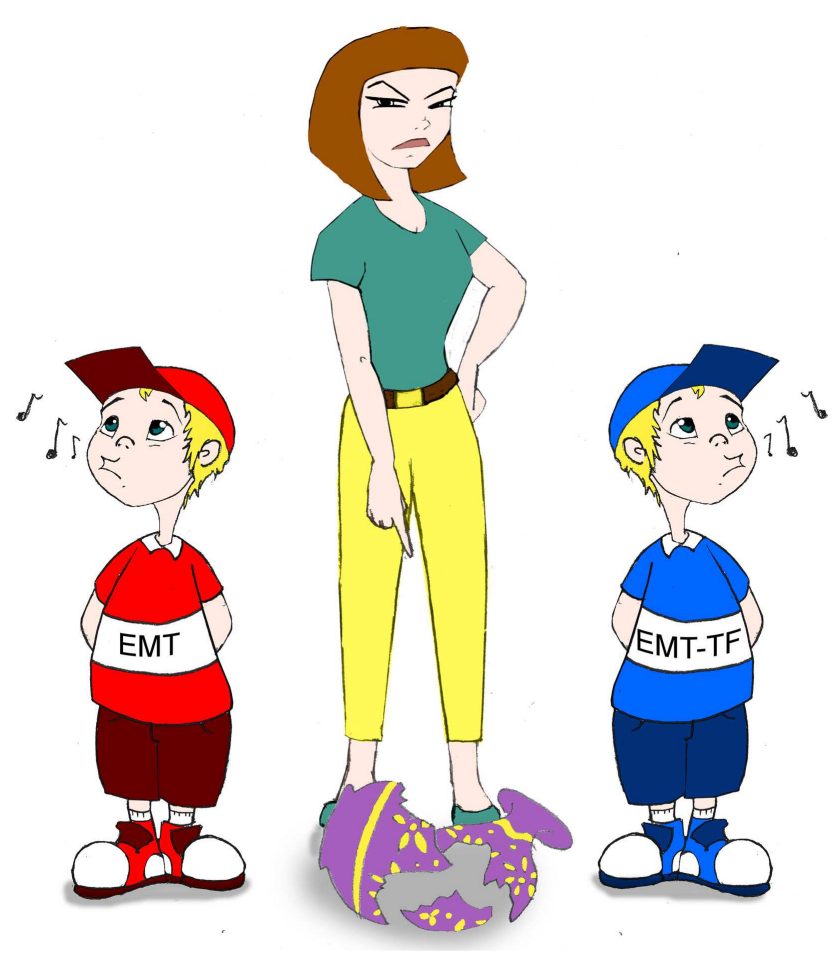

FIGURE 1 |As a mother trying to identify which of hers two sons broke the vase, scientists need to precisely determine the contribution of EMT and EMT-TFs in tumor development.

future, to be one of the main oncogenic functions of these embryonic transcription factors, with dedifferentiation being regularly associated with, and likely an integral part of, neoplastic transformation $(29,38$, 39). In this regard, the recent demonstration of a pivotal role of SNAIL1 in sarcomagenesis and its functions in preventing mesenchymal stem cell differentiation (20) likely reflects this behavior.

Resistance to therapeutic treatments in carcinoma cancer cells has also recurrently been associated with EMT. While this resistance might result from multiple mechanisms, including metabolic changes impacting on pro-drug activation and drug exclusion through transporters, recent observations also suggest that embryonic transcription factors might directly be involved in the emergence of such resistant cells, independently of their EMT-promoting functions, through various mechanisms. In a recent study, Zhang and co-workers have demonstrated that the ZEB1 transcription factor triggers radioresistance in an EMT-independent manner. Stabilized through phosphorylation by ATM, ZEB1 interacts with USP7 and enhances its ability to deubiquitinilate and stabilize CHK1, thereby favoring recombination-dependent DNA repair (40). In line with this observation, ZEB2 was shown to prevent ATM/ATR activation in response to a genotoxic stress in an EMT-independent manner and constitutes a factor of poor prognosis in bladder cancer patients treated with radiotherapy (41). TWIST1 was also previously demonstrated to trigger chemoresistance in an EMTindependent manner through its ability to induce AKT2 expression and to differently modulate the ratio between pro- and antiapoptotic members of the BCL-2 family [reviewed in Ref. (42)]. Lastly, SNAIL1 and SNAIL2 proteins protect kidney epithelial cells and hematopoietic precursor cells, respectively, from radiation-induced apoptosis by interfering with p53-target gene activation $(43,44)$.

The relative contribution of EMT and EMT inducers to tumor development is like the chicken and the egg question. Nonetheless, these observations collectively highlight numerous specific EMT-independent functions of these transcription factors, which likely merit consideration in line with the EMT-driven program that promotes carcinogenesis (Figure 1). This non-exhaustive list of functions of the EMT inducers likewise reflects only the emerged part of the iceberg. As previously mentioned, the EMT-promoting and failsafe program inhibition induced by ZEB1 requires different levels of protein expression (18). Furthermore, ZEB1 depletion in SNAIL1-expressing cells radio-sensitizes cells without affecting their commitment into an EMT process (40), likely unveiling a yet underestimated level of complexity. No doubt novel functions involving EMT-unrelated genetic programs induced in different cellular settings and protein expression levels will soon emerge as an additional oncogenic weapon of these factors. Their common denomination as EMT inducers will then be obsolete.

\section{REFERENCES}

1. Thiery JP, Acloque H, Huang RY, Nieto MA. Epithelial-mesenchymal transitions in development and disease. Cell (2009) 139:871-90. doi:10. 1016/j.cell.2009.11.007

2. Yang J, Weinberg RA. Epithelial-mesenchymal transition: at the crossroads of development and tumor metastasis. Dev Cell (2008) 14:818-29. doi: 10.1016/j.devcel.2008.05.009

3. De Craene B, Berx G. Regulatory networks defining EMT during cancer initiation and progression. Nat Rev Cancer (2013) 13:97-110. doi:10.1038/ nrc3447

4. Tran PT, Shroff EH, Burns TF, Thiyagarajan S, Das ST, Zabuawala T, et al. Twist1 suppresses senescence programs and thereby accelerates and maintains mutant Kras-induced lung tumorigenesis. PLoS Genet (2012) 8:e1002650. doi:10.1371/ journal.pgen.1002650

5. Dave N, Guaita-Esteruelas S, Gutarra S, Frias A, Beltran M, Peiro S, et al. Functional cooperation between Snaill and twist in the regulation of ZEB1 expression during epithelial to mesenchymal transition. J Biol Chem (2011) 286:12024-32. doi:10.1074/jbc.M110.168625

6. Maestro R, Dei Tos AP, Hamamori Y, Krasnokutsky S, Sartorelli V, Kedes L, et al. Twist is a potential oncogene that inhibits apoptosis. Genes Dev (1999) 13:2207-17. doi:10.1101/gad.13.17.2207

7. Valsesia-Wittmann S, Magdeleine M, Dupasquier S, Garin E, Jallas AC, Combaret V, et al. Oncogenic cooperation between $\mathrm{H}$-Twist and N-Myc overrides failsafe programs in cancer cells. Cancer Cell (2004) 6:625-30. doi:10.1016/j.ccr.2004.09.033

8. Ansieau S, Bastid J, Doreau A, Morel AP, Bouchet BP, Thomas C, et al. Induction of EMT by twist proteins as a collateral effect of tumor-promoting inactivation of premature senescence. Cancer Cell (2008) 14:79-89. doi:10.1016/j.ccr.2008.06.005

9. Morel AP, Hinkal GW, Thomas C, Fauvet F, Courtois-Cox S, Wierinckx A, et al. EMT inducers catalyze malignant transformation of mammary epithelial cells and drive tumorigenesis towards 
claudin-low tumors in transgenic mice. PLoS Genet (2012) 8:e1002723. doi:10.1371/journal. pgen. 1002723

10. Piccinin S, Tonin E, Sessa S, Demontis S, Rossi S, Pecciarini L, et al. A "twist box" code of p53 inactivation: twist box: p53 interaction promotes p53 degradation. Cancer Cell (2012) 22:404-15. doi:10.1016/j.ccr.2012.08.003

11. Burns TF, Dobromilskaya I, Murphy SC, Gajula RP, Thiyagarajan S, Chatley SN, et al. Inhibition of TWIST1 leads to activation of oncogene-induced senescence in oncogene-driven non-small cell lung cancer. Mol Cancer Res (2013) 11:329-38. doi:10. 1158/1541-7786.MCR-12-0456

12. Ohashi S, Natsuizaka M, Wong GS, Michaylira CZ, Grugan KD, Stairs DB, et al. Epidermal growth factor receptor and mutant p53 expand an esophageal cellular subpopulation capable of epithelial-tomesenchymal transition through ZEB transcription factors. Cancer Res (2010) 70:4174-84. doi: 10.1158/0008-5472.CAN-09-4614

13. Liu Y, El-Naggar S, Darling DS, Higashi Y, Dean DC. Zeb1 links epithelial-mesenchymal transition and cellular senescence. Development (2008) 135:579-88. doi:10.1242/dev.007047

14. Vega S, Morales AV, Ocana OH, Valdes F, Fabregat I, Nieto MA. Snail blocks the cell cycle and confers resistance to cell death. Genes Dev (2004) 18:1131-43. doi:10.1101/gad.294104

15. Franci C, Takkunen M, Dave N, Alameda F, Gomez S, Rodriguez R, et al. Expression of Snail protein in tumor-stroma interface. Oncogene (2006) 25:5134-44.

16. Spaderna S, Schmalhofer O, Hlubek F, Berx G, Eger A, Merkel S, et al. A transient, EMT-linked loss of basement membranes indicates metastasis and poor survival in colorectal cancer. Gastroenterology (2006) 131:830-40. doi:10.1053/j.gastro.2006. 06.016

17. Wu Y, Deng J, Rychahou PG, Qiu S, Evers BM, Zhou BP. Stabilization of snail by NF-kappaB is required for inflammation-induced cell migration and invasion. Cancer Cell (2009) 15:416-28. doi:10.1016/j.ccr.2009.03.016

18. Liu Y, Sanchez-Tillo E, Lu X, Huang L, Clem B, Telang $S$, et al. Sequential inductions of the ZEB1 transcription factor caused by mutation of $\mathrm{Rb}$ and then Ras proteins are required for tumor initiation and progression. J Biol Chem (2013) 288:11572-80. doi:10.1074/jbc.M112.434951

19. Tran DD, Corsa CA, Biswas H, Aft RL, Longmore GD. Temporal and spatial cooperation of Snaill and Twist1 during epithelial-mesenchymal transition predicts for human breast cancer recurrence. Mol Cancer Res (2011) 9:1644-57. doi:10.1158/ 1541-7786.MCR-11-0371

20. Alba-Castellon L, Batlle R, Franci C, FernandezAcenero MJ, Mazzolini R, Pena R, et al. Snaill expression is required for sarcomagenesis. Neoplasia (2014) 16:413-21. doi:10.1016/j.neo.2014.05. 002

21. Batlle R, Alba-Castellon L, Loubat-Casanovas J, Armenteros E, Franci C, Stanisavljevic J, et al. Snail1 controls TGF-beta responsiveness and differentiation of mesenchymal stem cells. Oncogene (2013) 32:3381-9. doi:10.1038/onc.2012.342
22. Rodriguez R, Rubio R, Menendez P. Modeling sarcomagenesis using multipotent mesenchymal stem cells. Cell Res (2012) 22:62-77. doi:10.1038/ cr.2011.157

23. Lee KE, Bar-Sagi D. Oncogenic KRas suppresses inflammation-associated senescence of pancreatic ductal cells. Cancer Cell (2010) 18:448-58. doi:10. 1016/j.ccr.2010.10.020

24. Dong C, Yuan T, Wu Y, Wang Y, Fan TW, Miriyala $\mathrm{S}$, et al. Loss of FBP1 by Snail-mediated repression provides metabolic advantages in basal-like breast cancer. Cancer Cell (2013) 23:316-31. doi:10.1016/ j.ccr.2013.01.022

25. Schieber MS, Chandel NS. ROS links glucose metabolism to breast cancer stem cell and EMT phenotype. Cancer Cell (2013) 23:265-7. doi:10. 1016/j.ccr.2013.02.021

26. Tsai JH, Donaher JL, Murphy DA, Chau S, Yang J. Spatiotemporal regulation of epithelialmesenchymal transition is essential for squamous cell carcinoma metastasis. Cancer Cell (2012) 22:725-36. doi:10.1016/j.ccr.2012.09.022

27. Ocana OH, Corcoles R, Fabra A, Moreno-Bueno $\mathrm{G}$, Acloque H, Vega S, et al. Metastatic colonization requires the repression of the epithelialmesenchymal transition inducer Prrx1. Cancer Cell (2012) 22:709-24. doi:10.1016/j.ccr.2012.10.012

28. Guo W, Keckesova Z, Donaher JL, Shibue T, Tischler V, Reinhardt F, et al. Slug and Sox9 cooperatively determine the mammary stem cell state. Cell (2012) 148:1015-28. doi:10.1016/j.cell.2012. 02.008

29. Mani SA, Guo W, Liao MJ, Eaton EN, Ayyanan A, Zhou AY, et al. The epithelial-mesenchymal transition generates cells with properties of stem cells. Cell (2008) 133:704-15. doi:10.1016/j.cell.2008.03. 027

30. Prat A, Perou CM. Deconstructing the molecular portraits of breast cancer. Mol Oncol (2011) 5:5-23. doi:10.1016/j.molonc.2010.11.003

31. Wellner U, Schubert J, Burk UC, Schmalhofer O, Zhu F, Sonntag A, et al. The EMT-activator ZEB1 promotes tumorigenicity by repressing stemnessinhibiting microRNAs. Nat Cell Biol (2009) 11:1487-95. doi:10.1038/ncb1998

32. Brabletz T, Jung A, Spaderna S, Hlubek F, Kirchner T. Opinion: migrating cancer stem cells - an integrated concept of malignant tumour progression. Nat Rev Cancer (2005) 5:744-9. doi:10.1038/ $\operatorname{nrc1694}$

33. Singh A, Greninger P, Rhodes D, Koopman L, Violette S, Bardeesy N, et al. A gene expression signature associated with "K-Ras addiction" reveals regulators of EMT and tumor cell survival. Cancer Cell (2009) 15:489-500. doi:10.1016/j.ccr.2009.03 022

34. Caramel J, Papadogeorgakis E, Hill L, Browne GJ, Richard G, Wierinckx A, et al. A switch in the expression of embryonic EMT-inducers drives the development of malignant melanoma. Cancer Cell (2013) 24:466-80. doi:10.1016/j.ccr.2013.08.018

35. Tulchinsky E, Pringle JH, Caramel J, Ansieau S. Plasticity of melanoma and EMT-TF reprogramming. Oncotarget (2014) 5:1-2.

36. Hoek KS, Goding CR. Cancer stem cells versus phenotype-switching in melanoma. Pigment Cell
Melanoma Res (2010) 23:746-59. doi:10.1111/j. 1755-148X.2010.00757.x

37. Denecker G, Vandamme N, Akay O, Koludrovic $\mathrm{D}$, Taminau J, Lemeire $\mathrm{K}$, et al. Identification of a ZEB2-MITF-ZEB1 transcriptional network that controls melanogenesis and melanoma progression. Cell Death Differ (2014) 21:1250-61. doi:10. 1038/cdd.2014.44

38. Morel AP, Lievre M, Thomas C, Hinkal G, Ansieau S, Puisieux A. Generation of breast cancer stem cells through epithelial-mesenchymal transition. PLoS One (2008) 3:e2888. doi:10.1371/journal. pone. 0002888

39. Scaffidi P, Misteli T. In vitro generation of human cells with cancer stem cell properties. Nat Cell Biol (2011) 13:1051-61. doi:10.1038/ncb2308

40. Zhang P, Wei Y, Wang L, Debeb BG, Yuan Y, Zhang J, et al. ATM-mediated stabilization of ZEB1 promotes DNA damage response and radioresistance through CHK1. Nat Cell Biol (2014) 16:864-75. doi: $10.1038 /$ ncb3013

41. Sayan AE, Griffiths TR, Pal R, Browne GJ, Ruddick A, Yagci T, et al. SIP1 protein protects cells from DNA damage-induced apoptosis and has independent prognostic value in bladder cancer. Proc Natl Acad Sci U S A (2009) 106:14884-9. doi:10.1073/pnas.0902042106

42. Ansieau S, Morel AP, Hinkal G, Bastid J, Puisieux A. TWISTing an embryonic transcription factor into an oncoprotein. Oncogene (2010) 29:3173-84. doi:10.1038/onc.2010.92

43. Wu WS, Heinrichs S, Xu D, Garrison SP, Zambetti GP, Adams JM, et al. Slug antagonizes p53mediated apoptosis of hematopoietic progenitors by repressing puma. Cell (2005) 123:641-53. doi: 10.1016/j.cell.2005.09.029

44. Escriva M, Peiro S, Herranz N, Villagrasa P, Dave N, Montserrat-Sentis B, et al. Repression of PTEN phosphatase by Snaill transcriptional factor during gamma radiation-induced apoptosis. $\mathrm{Mol}$ Cell Biol (2008) 28:1528-40. doi:10.1128/MCB. 02061-07

Conflict of Interest Statement: The authors declare that the research was conducted in the absence of any commercial or financial relationships that could be construed as a potential conflict of interest.

Received: 30 September 2014; accepted: 26 November 2014; published online: 16 December 2014. Citation: Ansieau S, Collin G and Hill L (2014) EMT or EMT-promoting transcription factors, where to focus the light? Front. Oncol. 4:353. doi: 10.3389/fonc.2014.00353 This article was submitted to Molecular and Cellular Oncology, a section of the journal Frontiers in Oncology. Copyright (c) 2014 Ansieau, Collin and Hill. This is an open-access article distributed under the terms of the Creative Commons Attribution License (CC BY). The use, distribution or reproduction in other forums is permitted, provided the original author(s) or licensor are credited and that the original publication in this journal is cited, in accordance with accepted academic practice. No use, distribution or reproduction is permitted which does not comply with these terms. 